\title{
Transurethral Procedures for Lower Urinary Tract Symptoms Resulting From Benign Prostatic Enlargement: A Quality and Meta-Analysis
} \author{
Hee Jong Jeong ${ }^{7}$, Jae Hyun Bae ${ }^{8}$, Young-Suk Lee ${ }^{9}$, Joon Chul Kim ${ }^{10}$ \\ Department of Urology, Hanyang University College of Medicine, Seoul; \\ ${ }^{1}$ Department of Urology, Ajou University School of Medicine, Suwon; \\ ${ }^{2}$ Department of Urology, Samsung Medical Center, Sungkyunkwan University School of Medicne, Seoul; \\ ${ }^{3}$ Department of Urology, Chung-Ang University College of Medicine, Seoul; \\ ${ }^{4}$ Department of Urology, SMG-SNU Boramae Medical Center, Seoul; \\ ${ }^{5}$ Department of Urology, Veterans Health Service Medical Center, Seoul; \\ ${ }^{6}$ Department of Urology, Seoul National University Hospital, Seoul; \\ ${ }^{7}$ Department of Urology, Wonkwang University School of Medicine \& Hospital, Iksan; \\ ${ }^{8}$ Department of Urology, Korea University College of Medicine, Seoul; \\ ${ }^{9}$ Department of Urology, Samsung Changwon Hospital, Sungkyunkwan University School of Medicine, Changwon; \\ ${ }^{10}$ Department of Urology, The Catholic University College of Medicine, Seoul, Korea
}

Seung Wook Lee, Jong Bo Choi ${ }^{1}$, Kyu-Sung Lee, Tae-Hyoung Kim³, Hwancheol Son ${ }^{4}$, Tae Young Jung ${ }^{5}$, Seung-June $\mathrm{Oh}^{6}$,

\begin{abstract}
Purpose: Thanks to advancements in surgical techniques and instruments, many surgical modalities have been developed to replace transurethral resection of the prostate (TURP). However, TURP remains the gold standard for the surgical treatment of benign prostatic hyperplasia (BPH). We conducted a meta-analysis on the efficacy and safety of minimally invasive surgical therapies for BPH compared with TURP.

Methods: This meta-analysis used a Medline search assessing the period from 1997 to 2011. A total of 784 randomized controlled trials were identified in an electronic search. Among the 784 articles, 36 randomized controlled trials that provided the highest level of evidence (level $1 \mathrm{~b}$ ) were included in the meta-analysis. We also conducted a quality analysis of selected articles.

Results: Only 2 articles (5.56\%) were assessed as having a low risk of bias by use of the Cochrane collaboration risk of bias tool. On the other hand, by use of the Jadad scale, there were 26 high-quality articles $(72.22 \%)$. Furthermore, 28 articles (77.78\%) were assessed as high-quality articles by use of the van Tulder scale. Holmium laser enucleation of the prostate (HoLEP) showed the highest reduction of the International Prostate Symptom Score compared with TURP $(\mathrm{P}<0.0001)$. Bipolar TURP, bipolar transurethral vaporization of the prostate, HoLEP, and open prostatectomy showed superior outcome in postvoid residual urine volume and maximum flow rate. The intraoperative complications of the minimally invasive surgeries had no statistically significant inferior outcomes compared with TURP. Also, there were no statistically significant differences in any of the modalities compared with TURP.

Conclusions: The selection of an appropriate surgical modality for BPH should be assessed by fully understanding each patient's clinical conditions.
\end{abstract}

Keywords: Prostatic hyperplasia; Holmium; Lasers; Potassium titanylphosphate; Transurethral resection of prostate; Meta-analysis

\section{INTRODUCTION}

Benign prostatic hyperplasia (BPH), which causes lower uri- nary tract symptoms (LUTS), is one of the most common diseases of aging men [1]. LUTS can reduce quality of life by impeding normal activities and causing complications such as acute
Corresponding author: Joon Chul Kim

Department of Urology, Bucheon St. Mary's Hospital,

The Catholic University of Korea College of Medicine, 327 Sosa-ro 327beon-gil,

Wonmi-gu, Bucheon 420-717, Korea

Tel: +82-32-340-7071 / Fax: +82-32-340-2124 / E-mail: kjc@catholic.ac.kr

Submitted: March 25, 2013 / Accepted after revision: May 2, 2013
This is an Open Access article distributed under the terms of the Creative Commons Attribution Non-Commercial License (http://creativecommons.org/licenses/by-nc/3.0/) which permits unrestricted non-commercial use, distribution, and reproduction in any medium, provided the original work is properly cited. 
urinary retention or urinary tract infection. $\mathrm{BPH}$ is histologically observed in about half of men in their 60s and in most men aged 80 and older [2]. Nowadays, various medications are used to treat LUTS resulting from BPH (LUTS/BPH); these include 5-alpha-reductase inhibitors (5-ARIs), alpha-adrenergic blockers, and others. Moreover, many surgical treatment methods have been introduced, such as resection or enucleation.

Currently, the gold standard surgical treatment for LUTS/BPH is transurethral resection of the prostate (TURP) [3,4]. However, TURP is associated with complications including bleeding, pain, infection, urethral stricture, bladder neck contraction, erectile dysfunction, incontinence, and retrograde ejaculation [5]. Therefore, many endoscopic surgical methods have been suggested to replace TURP as the new standard [6]. There has been a continuous rise in the use of minimally invasive surgical therapies for LUTS/BPH, including bipolar TURP, bipolar transurethral vaporization of the prostate (TUVP), holmium laser enucleation of the prostate (HoLEP), and potassium-titanyl-phosphate (KTP) laser vaporization of the prostate. So far, numerous articles have reported on comparisons of these new techniques with TURP. Ahyai et al. [6] reported a meta-analysis on functional outcomes and complications of transurethral prostatectomy for LUTS/BPH. According to those authors, many minimally invasive surgical therapies for LUTS/BPH showed statistically comparable efficacy and overall morbidity to TURP. However, that report included randomized controlled trials published from 1997 to 2009.

Therefore, we conducted a meta-analysis on the efficacy and safety of minimally invasive surgical therapies for LUTS/BPH compared with TURP by analyzing more recent articles that were published from 2010 to 2011. In addition, we assessed the quality of these articles by using the Jadad scale, the van Tulder scale (VTS), and Cochrane collaboration risk of bias tool (CCRBT).

\section{MATERIALS AND METHODS}

\section{Searching Strategy}

This meta-analysis used a Medline search assessing the period from 1997 to 2011. We searched published articles by using MeSH phrases such as "benign prostatic hyperplasia," "enlargement," and "obstruction"; "minimally invasive surgical therapy"; "randomized controlled trial [Publication Type]"; and the specific TURP name. There were no limitations on languages.

\section{Study Selection}

A total of 784 randomized controlled trials were identified in an electronic search. Among the 784 articles, 36 randomized controlled trials that provided the highest level of evidence (level 1b) were included in the meta-analysis. Studies that were not randomized or that had no comparator were excluded.

\section{Data Extraction}

We collected the following data: comparator; name of first author; year of publication; number of patients in each group; follow-up period; baseline data, including age, prostate volume $\left(\mathrm{cm}^{3}\right)$, International Prostate Symptom Score (IPSS), quality of life (QoL) score, postvoiding residual urine volume (PVR; $\mathrm{mL}$ ), and maximum flow rate $(\mathrm{Qmax} ; \mathrm{mL} / \mathrm{sec})$ before the procedure (Table 1); perioperative outcomes, including operative time ( $\mathrm{min}$ ), weight of resected tissue (g), and length of catheter use (day); functional outcomes, including IPSS and Qmax after the surgery; and complications (Table 2).

\section{Quality Analysis}

We assessed the quality of the selected articles by using the Jadad scale, VTS, and CCRBT [7]. All quality assessments of articles were performed by two reviewers. If there were different outcomes, the two reviewers and a third reviewer resolved the discrepancy in the results through discussion.

\section{Statistical Analysis}

The primary endpoint of the present analysis was functional outcomes, including IPSS/QoL and Qmax/PVR; perioperative results, including operative time ( $\mathrm{min}$ ) and length of catheter use (day); and incidence rate of complications, including bleeding, blood transfusion, conversion to TURP, capsule perforation, transurethral resection syndrome, acute urinary retention, clot retention, secondary apical resection, secondary coagulation revision, secondary bleeding, infection, urethral stricture, bladder neck stenosis, urgency, stress urinary incontinence, and reoperation/intervention requirement. Pooled odds ratios and 95\% confidence intervals were calculated for the dichotomous and continuous outcome data between the various operative methods and TURP, respectively. The Q-statistic was used to analyze heterogeneity [8]. If I $2>50 \%$, we considered it as heterogeneous and a random effect model was performed. IBM SPSS ver. 18.0 (IBM Co., Armonk, NY, USA) and SAS ver. 9.1.3 (SAS Institute, Cary, NC, USA) were used for the statistical analysis. All tests were two-sided, with a significance level of 0.05 . 
Table 1. Summary of (mean) baseline characteristics from included randomised trials comparing minimally invasive therapies with transurethral resection of the prostate

\begin{tabular}{|c|c|c|c|c|c|c|c|c|c|c|}
\hline $\begin{array}{l}\text { Publication } \\
\text { year }\end{array}$ & Author ${ }^{\text {a) }}$ & Comparator & $\begin{array}{c}\text { No. of } \\
\text { patients }\end{array}$ & $\begin{array}{l}\text { Follow-up } \\
(\mathrm{yr})\end{array}$ & $\begin{array}{l}\text { Age } \\
(\mathrm{yr})\end{array}$ & $\begin{array}{c}\text { Prostate } \\
\text { volume }\left(\mathrm{cm}^{3}\right)\end{array}$ & IPSS & QoL & $\begin{array}{c}\text { Qmax } \\
(\mathrm{mL} / \mathrm{sec})\end{array}$ & $\begin{array}{l}\text { PVR } \\
(\mathrm{mL})\end{array}$ \\
\hline 2011 & Geavlete et al. [S-1] & BPVPvs. TURis & $170 ; 170 ; 170$ & 1.5 & N/A & $54.1 ; 53.7 ; 54.8$ & $24.3 ; 24.0 ; 24.2$ & $4.3 ; 4.5 ; 4.3$ & $6.6 ; 6.1 ; 6.4$ & $91 ; 96 ; 88$ \\
\hline 2011 & Fagerstroml et al. [S-2] & Bipolar TURP & $90 ; 95 ; 185$ & 1.5 & $69.5 ; 72.7$ & $55.6 ; 58.2$ & $21.7 ; 20.4$ & $3.9 ; 3.7$ & N/A & N/A \\
\hline 2011 & Fayad et al. [S-3] & HoLEP vs. Bipolar TURP & $30 ; 30$ & 0.5 & $60.0 ; 61.2$ & $76.5 ; 80.6$ & $22.6 ; 22.2$ & N/A & $7.4 ; 6.9$ & N/A \\
\hline 2010 & Chen et al. [S-4] & Bipolar TURP (TURis) & $50 ; 50$ & 2.0 & $69.7 ; 71.2$ & $60.2 ; 59.1$ & $22.8 ; 21.8$ & N/A & $7.1 ; 7.9$ & $73.1 ; 80$ \\
\hline 2010 & Zhao et al. [S-5] & Plasmakinetic enucleation & $102 ; 102$ & 3.0 & $67.3 ; 67.8$ & $69.2 ; 67.5$ & $23.2 ; 22.4$ & $4.5 ; 4.8$ & $8.3 ; 8.0$ & $92 ; 97$ \\
\hline 2010 & Al-Ansari et al. [S-6] & KTP & $60 ; 60$ & 3.0 & $66.3 ; 67.1$ & $61.8 ; 60.3$ & $27.2 ; 27.9$ & N/A & $6.9 ; 6.4$ & $53.2 ; 57$ \\
\hline 2010 & Simforoosh et al. [S-7] & Open & $51 ; 49$ & 1.0 & $71.7 ; 61.0$ & $47.9 ; 44.4$ & $27.1 ; 27.1$ & N/A & $7.0 ; 8.1$ & $62 ; 47$ \\
\hline 2010 & Ou et al. [S-8] & Open & $34 ; 35$ & 1.0 & $71.3 ; 70.9$ & $138.4 ; 131.0$ & $23.1 ; 21.7$ & $4.4 ; 4.3$ & $5.0 ; 6.2$ & $80.3 ; 92.7$ \\
\hline 2010 & Elmansy et al. [S-9] & HoLEP vs. PVP & $57 ; 52$ & 4.0 & $72.7 ; 71.6$ & $33.1 ; 37.3$ & $20 ; 18.4$ & $3.8 ; 3.6$ & $6.7 ; 6.4$ & $205 ; 215$ \\
\hline 2008 & Horasanli et al. [S-10] & KTP & $39 ; 37$ & 0.5 & $69.2 ; 68.3$ & $86 ; 88$ & $18.9 ; 20.2$ & N/A & $8.6 ; 9.2$ & $183.0 ; 176.9$ \\
\hline 2008 & Alivizatos et al. [S-11] & KTP vs. open & $65 ; 60$ & 1.0 & $74.0 ; 67.5$ & $93.0 ; 96.0$ & $20.0 ; 21.0$ & $3.0 ; 3.0$ & $8.6 ; 8.0$ & $97.0 ; 89.0$ \\
\hline 2008 & Iori et al. [S-12] & Bipolar TURP & $26 ; 25$ & 1.0 & $65.0 ; 63.0$ & $49.0 ; 48.0$ & $21.0 ; 20.0$ & $3.0 ; 3.6$ & $7.0 ; 8.7$ & $99.0 ; 96.0$ \\
\hline $2008 ; 2002$ & Kuntz and Lehrich [S-13,14] & HoLEP vs. open & $60 ; 60$ & 5.0 & $69.2 ; 71.2$ & $114.6 ; 113.0$ & $22.1 ; 21.0$ & N/A & $3.8 ; 3.6$ & 280 \\
\hline 2007 & Michielsen et al. [S-15] & Bipolar TURP & $118 ; 120$ & 1.5 & $73.8 ; 73.1$ & N/A & N/A & N/A & N/A & N/A \\
\hline $2007 ; 2006$ & de Sio et al. [S-16] & Bipolar TURP & $35 ; 35$ & 3.0 & $59.0 ; 61.0$ & $51.6 ; 47.5$ & $24.2 ; 24.3$ & $4.2 ; 3.9$ & $7.1 ; 6.9$ & $80.0 ; 75.0$ \\
\hline 2007 & Ho et al. [S-17] & Bipolar TURP & $48 ; 52$ & 1.0 & $66.6 ; 66.5$ & $56.5 ; 54.8$ & $22.6 ; 24.6$ & N/A & $6.8 ; 6.5$ & N/A \\
\hline 2007 & Kaya et al. [S-18] & Bipolar TUVP & $25 ; 15$ & 3.0 & $67.2 ; 66.0$ & $50.0 ; 51.0$ & $21.0 ; 22.0$ & N/A & $6.0 ; 6.0$ & N/A \\
\hline \multirow[t]{2}{*}{$2007 ; 2004$} & Kuntz and Lehrich [S-19]/ & HoLEP & $100 ; 100$ & 3.0 & $68.0 ; 68.7$ & $53.5 ; 49.9$ & $22.1 ; 21.4$ & N/A & $45.9 ; 5.9$ & $238.0 ; 216.0$ \\
\hline & Ahyai and Leihrich [S-20] & & & & & & & & & \\
\hline 2006 & Patankar et al. [S-21] & Bipolar TURP & $53 ; 51$ & 0.1 & $64.0 ; 62.0$ & $51.3 ; 52.3$ & $23.3 ; 23.7$ & N/A & $5.9 ; 6.4$ & N/A \\
\hline 2006 & Nuhoglu et al. [S-22] & Bipolar TURP & $27 ; 30$ & 1.0 & $64.6 ; 65.2$ & $47.0 ; 49.0$ & $17.6 ; 17.3$ & N/A & $6.9 ; 7.3$ & $96.0 ; 88.0$ \\
\hline 2006 & Gupta et al. [S-23] & HoLEP & $50 ; 50$ & 1.0 & $65.8 ; 65.7$ & $57.9 ; 59.8$ & $23.4 ; 23.3$ & N/A & $5.2 ; 4.5$ & $112.0 ; 84.0$ \\
\hline 2006 & Neill et al. [S-24] & HoLEP & $30 ; 30$ & 2.0 & $71.7 ; 70.3$ & $77.8 ; 70.0$ & $26.0 ; 23.7$ & $4.8 ; 4.7$ & $8.4 ; 8.3$ & N/A \\
\hline 2006 & Naspro et al. [S-25] & HoLEP vs. open & $41 ; 39$ & 2.0 & $66.3 ; 67.3$ & $113.3 ; 124.2$ & $20.1 ; 21.6$ & $4.1 ; 4.4$ & $7.8 ; 8.3$ & N/A \\
\hline 2006 & Bouchiers-Hayes et al. [S-26] & KTP & $38 ; 38$ & 1.0 & $65.2 ; 66.2$ & $42.4 ; 33.2$ & N/A & N/A & N/A & $147.0 ; 119.0$ \\
\hline 2006 & Hon et al. [S-27] & Bipolar TUVP & $81 ; 79$ & 0.7 & $66.1 ; 68.1$ & $38.0 ; 40.0$ & $21.3 ; 20.6$ & $4.2 ; 4.3$ & $12.0 ; 11.9$ & $147.0 ; 182.0$ \\
\hline 2005 & Singh et al. [S-28] & Bipolar TURP & $30 ; 30$ & 0.3 & $68.9 ; 67.9$ & $24.1 ; 27.9$ & $20.5 ; 21.6$ & $4.6 ; 4.4$ & $5.8 ; 5.1$ & $124.0 ; 136.0$ \\
\hline 2005 & Seckiner et al. [S-29] & Bipolar TURP & $24 ; 24$ & 1.0 & $61.2 ; 63.9$ & $49.4 ; 41.4$ & $24.1 ; 23.2$ & $4.4 ; 4.7$ & $8.5 ; 8.3$ & $88.0 ; 138.0$ \\
\hline 2005 & Fung et al. [S-30] & Bipolar TURP & $21 ; 30$ & 0.3 & $72.5 ; 73.0$ & N/A & $15.8 ; 19.4$ & $3.6 ; 3.6$ & N/A & N/A \\
\hline 2005 & Tefekli et al. [S-31] & Bipolar TUVP & $51 ; 50$ & 1.0 & $68.7 ; 69.4$ & $50.1 ; 50.4$ & $21.3 ; 20.4$ & N/A & $7.8 ; 8.3$ & N/A \\
\hline 2004 & Yang et al. [S-32] & Bipolar TURP & $58 ; 59$ & 0.3 & N/A & $46 ; 49$ & $20.9 ; 21.6$ & $3.7 ; 4.0$ & $10.4 ; 10.9$ & $99.0 ; 150$ \\
\hline 2004 & Montorsi et al. [S-33] & HoLEP & $52 ; 48$ & 1.0 & $65.1 ; 64.5$ & $70.3 ; 56.2$ & $21.6 ; 21.9$ & $4.6 ; 4.7$ & $8.2 ; 7.8$ & N/A \\
\hline 2003 & Dumsumir et al. [S-34] & Bipolar TUVP & $30 ; 21$ & 1.0 & $63.0 ; 60.0$ & $36.0 ; 42.0$ & $24.0 ; 17.0$ & N/A & $9.6 ; 10.4$ & $112.0 ; 96.0$ \\
\hline
\end{tabular}

IPSS, International Prostate Symptom Score; QoL, quality of life; Qmax, maximum flow rate; PVR, postvoid residual urine volume; BPVP, bipolar plasma vaporization of the prostate; TURis, transurethral resection of prostate in saline; N/A, not available; TURP, transurethral resection of prostate; HoLEP, holmium laser enucleation of the prostate; KTP, potassium-titanyl-phosphate; PVP, plasma vaporization of the prostate; TUVP, transurethral vaporisation of the prostate.

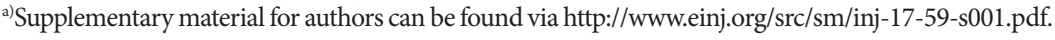

\section{RESULTS}

\section{Quality Analysis of Selected Articles}

Among the 36 articles, only 2 articles (5.56\%) were assessed as having a low risk of bias, 7 articles (19.44\%) were assessed as having a moderate risk of bias, and 27 articles (0.75\%) were evaluated as having a high risk of bias by use of the CCRBT. On the other hand, by use of the Jadad scale, there were 26 high-quality arti- cles (72.22\%). Furthermore, 28 articles (77.78\%) were assessed as high-quality articles by use of the VTS.

\section{Meta-Analysis of Functional Outcomes IPSS (Fig. 1) and QoL (Fig. 2)}

Only KTP laser vaporization of the prostate showed an inferior outcome compared with TURP ( $\mathrm{P}=0.0272)$. Otherwise, HoLEP showed the highest reduction of the IPSS compared with TURP 
Table 2. Summary of (mean) outcome data from included randomised studies comparing minimally invasive surgical therapies with transurethral resection of the prostate

\begin{tabular}{|c|c|c|c|c|c|c|c|c|}
\hline Author ${ }^{\text {a) }}$ & Comparator & $\begin{array}{l}\text { Operating time } \\
\qquad(\min )\end{array}$ & $\begin{array}{c}\text { Resected } \\
\text { tissue (gm) }\end{array}$ & $\begin{array}{l}\text { Catheterization } \\
\text { time (day) }\end{array}$ & IPSS & QoL & $\begin{array}{c}\text { Qmax } \\
(\mathrm{mL} / \mathrm{sec})\end{array}$ & $\begin{array}{l}\text { PVR } \\
(\mathrm{mL})\end{array}$ \\
\hline Geavlete et al. [S-1] & BPVP vs. TURis vs. TURP & $39.7 ; 52.1 ; 55.6$ & N/A & $0.9 ; 1.9 ; 3.0$ & $5.0 ; 7.9 ; 8.3$ & $1.0 ; 1.3 ; 1.5$ & $23.7 ; 20.6 ; 20.2$ & $29 ; 31 ; 33$ \\
\hline Fagerstroml et al. [S-2] & Bipolar TURP & $62 ; 66$ & $27.3 ; 26.3$ & $0.8 ; 0.8$ & $7.1 ; 7.6$ & $0.9 ; 1.1$ & N/A & N/A \\
\hline Fayad et al. [S-3] & HoLEP vs. Bipolar TURP & $110.5 ; 76.5$ & $55.9 ; 65.6$ & N/A & $5.5 ; 5.3$ & N/A & $20.8 ; 20.5$ & $20.3 ; 25.6$ \\
\hline Chen et al. [S-4] & Bipolar TURP (TURis) & $59 ; 60$ & $40 ; 38.9$ & N/A & $3.7 ; 3.8$ & N/A & $25.5 ; 24.8$ & N/A \\
\hline Zhao et al. [S-5] & Plasmakinetic enucleation & $62.8 ; 55.3$ & $56.4 ; 43.8$ & $2.2 ; 3.4$ & $2.4 ; 4.3$ & $0.6 ; 1.6$ & $28.8 ; 25.1$ & $5.0 ; 5.4$ \\
\hline Al-Ansari et al. [S-6] & КTP & $89 ; 80$ & N/A & $1.4 ; 2.7$ & $10.9 ; 9.2$ & N/A & $17.2 ; 20$ & $12.5 ; 10.5$ \\
\hline Simforoosh et al. [S-7] & Open & N/A & $34.5 ; 31.0$ & $7 ; 5$ & $4.8 ; 6.7$ & $2.3 ; 2.8$ & $18.1 ; 16.1$ & $2.0 ; 2.5$ \\
\hline Ou et al. [S-8] & Open & $109.5 ; 103.7$ & $116.8 ; 69.7$ & $7.5 ; 4.1$ & $2.8 ; 8.1$ & $1.26 ; 2.5$ & $16.4 ; 12.5$ & $9.12 ; 27.3$ \\
\hline Elmansy et al. [S-9] & HoLEP vs. PVP & $69.8 ; 55.5$ & N/A & $2.1 ; 1.6$ & $5.9 ; 6.6$ & $1.1 ; 1.2$ & $17.7 ; 18.5$ & $38.8 ; 43.9$ \\
\hline Horasanli et al. [S-10] & КTP & $87 ; 51$ & N/A & $1.7 ; 3.9$ & $13.1 ; 6.4$ & N/A & $13.3 ; 20.7$ & $79 ; 23$ \\
\hline Alivizatos et al. [S-11] & KTP vs. Open & $80.0 ; 50.0$ & $0.0 ; 73.5$ & $1.0 ; 5.0$ & $9.0 ; 8.0$ & $1.0 ; 1.0$ & $16.0 ; 15.1$ & $17 ; 12$ \\
\hline Iori et al. [S-12] & Bipolar TURP & $39.1 ; 31.7$ & N/A & $1.0 ; 1.3$ & $7.0 ; 6.7$ & $1.0 ; 1.0$ & $24.2 ; 23.2$ & $20.0 ; 27.0$ \\
\hline Kuntz and Lehrich [S-13,14] & HoLEP vs. Open & $135.9 ; 90.6$ & $93.7 ; 96.4$ & $1.8 ; 8.1$ & $3.0 ; 3.2$ & N/A & $24.3 ; 24.4$ & $10.6 ; 5.3$ \\
\hline Michielsen et al. [S-15] & Bipolar TURP & $56.0 ; 44.0$ & $21.0 ; 21.3$ & $4.0 ; 4.5$ & N/A & N/A & N/A & N/A \\
\hline de Sio et al. [S-16] & Bipolar TURP & $49.0 ; 53.0$ & $20.0 ; 24.0$ & $3.0 ; 4.2$ & $6.8 ; 6.2$ & $1.0 ; 0.8$ & $20.5 ; 21.5$ & $25.0 ; 20.0$ \\
\hline Ho et al. [S-17] & Bipolar TUVP & N/A & N/A & N/A & $7.6 ; 14.4$ & N/A & $5.7 ; 21.8$ & N/A \\
\hline Kaya et al. [S-18] & HoLEP & $94.6 ; 73.8$ & $35.9 ; 37.2$ & $1.0 ; 2.0$ & $3.0 ; 10.0$ & N/A & $29.0 ; 27.5$ & $8 ; 20$ \\
\hline Kuntz and Lehrich [S-19]/ & Bipolar TURP & $50.0 ; 57.9$ & N/A & $0.8 ; 1.8$ & $6.1 ; 7.7$ & N/A & $19.2 ; 20.7$ & N/A \\
\hline \multicolumn{9}{|l|}{ Ahyai and Leihrich [S-20] } \\
\hline Patankar et al. [S-21] & Bipolar TURP & $55.0 ; 52.0$ & N/A & $1.8 ; 3.2$ & $5.4 ; 4.7$ & N/A & $17.1 ; 17.9$ & N/A \\
\hline Nuhoglu et al. [S-22] & HoLEP & $75.4 ; 62.6$ & $17.2 ; 24.2$ & $1.2 ; 2.0$ & $5.2 ; 5.6$ & N/A & $25.1 ; 23.7$ & $20.0 ; 20.0$ \\
\hline Gupta et al. [S-23] & HoLEP & $62.1 ; 33.1$ & $40.4 ; 24.7$ & $0.7 ; 1.9$ & $6.1 ; 5.2$ & $1.25 ; 1.3$ & $21.0 ; 19.0$ & $33.7 ; 51.8$ \\
\hline Neill et al. [S-24] & HoLEP vs. Open & $72.1 ; 58.3$ & $59.3 ; 87.9$ & $1.5 ; 4.1$ & $7.9 ; 8.1$ & $1.5 ; 1.66$ & $19.2 ; 20.1$ & N/A \\
\hline Naspro et al. [S-25] & KTP & $31.3 ; 30.2$ & N/A & $0.51 ; 1.9$ & $12.0 ; 12.4$ & N/A & $19.9 ; 20.6$ & $37 ; 27$ \\
\hline Bouchiers-Hayes et al. [S-26] & Bipolar TUVP & $32.6 ; 28.5$ & $0.0 ; 21.5$ & N/A & $7.7 ; 6.9$ & $1.7 ; 1.5$ & $25.6 ; 23.5$ & $64.0 ; 69.0$ \\
\hline Hon et al. [S-27] & Bipolar TURP & $39.3 ; 36.9$ & $24.0 ; 27.6$ & $2.5 ; 3.4$ & $5.3 ; 6.2$ & $1.1 ; 1.0$ & $19.0 ; 17.8$ & N/A \\
\hline Singh et al. [S-28] & Bipolar TURP & $52.9 ; 52.9$ & $36.6 ; 31.9$ & $3.1 ; 3.1$ & $8.7 ; 8.3$ & $1.8 ; 2.0$ & $18.8 ; 15.7$ & N/A \\
\hline Seckiner et al. [S-29] & Bipolar TURP & $36.6 ; 32.9$ & $18.6 ; 25.1$ & $1.1 ; 1.2$ & $7.0 ; 9.7$ & N/A & $16.6 ; 14.7$ & N/A \\
\hline Fung et al. [S-30] & Bipolar TUVP & $40.3 ; 57.8$ & N/A & $2.3 ; 3.8$ & $7.9 ; 7.3$ & N/A & $17.2 ; 16.9$ & N/A \\
\hline Tefekli et al. [S-31] & Bipolar TURP & $46.0 ; 55.0$ & N/A & $2.7 ; 3.2$ & $10.8 ; 11.1$ & $2.1 ; 2.2$ & $17.1 ; 14.8$ & $13.9 ; 34.5$ \\
\hline Yang et al. [S-32] & HoLEP & $74.0 ; 57.0$ & $36.1 ; 25.4$ & $1.3 ; 2.4$ & $4.1 ; 3.9$ & $1.4 ; 0.8$ & $25.1 ; 24.7$ & N/A \\
\hline Montorsi et al. [S-33] & Bipolar TUVP & $33.0 ; 26.0$ & $0.0 ; 8.0$ & $0.8 ; 0.7$ & $6.0 ; 5.0$ & N/A & $18.0 ; 16.0$ & $90.0 ; 80.0$ \\
\hline
\end{tabular}

IPSS, International Prostate Symptom Score; QoL, quality of life; Qmax, maximum flow rate; PVR, postvoid residual urine volume; BPVP, bipolar plasma vaporization of the prostate; TURis, transurethral resection of prostate in saline; TURP, transurethral resection of prostate; N/A, not available; HoLEP, holmium laser enucleation of the prostate; KTP, potassium-titanyl-phosphate; PVP, plasma vaporization of the prostate; TUVP, transurethral vaporisation of the prostate.

a) Supplementary material for authors can be found via http://www.einj.org/src/sm/inj-17-59-s001.pdf.

$(\mathrm{P}<0.0001)$. Moreover, only HoLEP showed a higher QoL, although it was not statistically significant $(\mathrm{P}=0.1252)$.

\section{Maximum flow rate (Fig. 3) and postvoid residual urine volume (Fig. 4)}

Only KTP showed an inferior outcome in Qmax and PVR (P= 0.1407, $\mathrm{P}=0.0058)$. However, bipolar TURP, bipolar TUVP, HoLEP, and open prostatectomy showed a superior outcome in
Qmax and PVR. Open prostatectomy and HoLEP could not be statistically analyzed for PVR owing to insufficient data.

\section{Meta-Analysis of Complications Of Surgery Intraoperative complications (Fig. 5)}

No surgical methods showed statistically significant inferior outcomes compared with TURP. However, HoLEP showed the highest complication rate $(\mathrm{P}=0.0710)$ and KTP showed a sta- 
tistically significantly lower complication rate than TURP $(\mathrm{P}<$ 0.0001). HoLEP showed the highest incidence of bladder mucosal injury, and TURP showed the highest incidence of intraoperative transfusion (Table 3).

\section{Perioperative complications (Fig. 6)}

KTP and HoLEP showed statistically significantly inferior outcomes compared with TURP $(\mathrm{P}<0.0001, \mathrm{P}=0.0342$, respectively). Acute urinary retention, secondary apical resection, and febrile urinary tract infection occurred most commonly with KTP. TURP showed the highest occurrence rate of clot retention and hematuria episodes (Table 4).

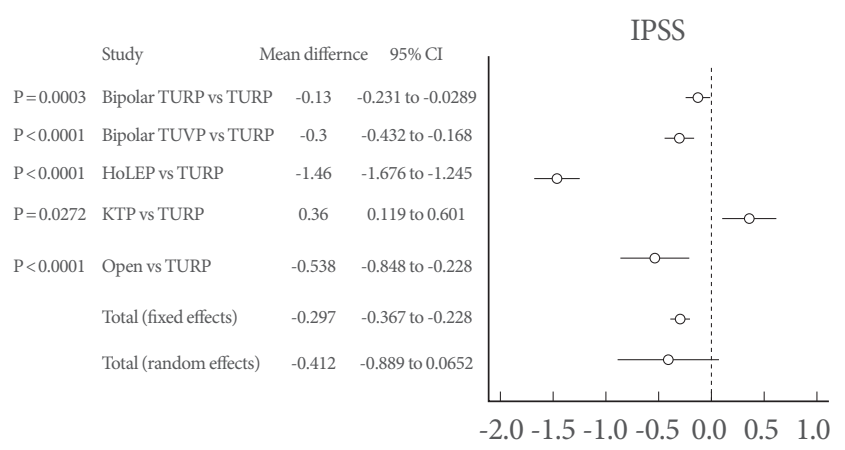

Fig. 1. Forest plot of International Prostate Symptom Score (IPSS). CI, confidence interval; TURP, transurethral resection of the prostate; IPSS, International Prostate Symptom Score; TUVP, transurethral vaporization of the prostate; HoLEP, holmium laser enucleation of the prostate; KTP, potassium-titanyl-phosphate.

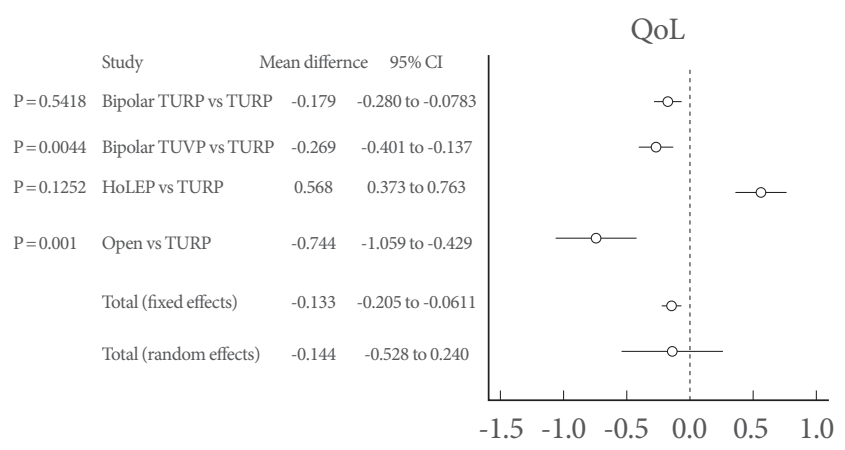

Fig. 2. Forest plot of quality of life (QoL, question 8 of the IPSS). $\mathrm{CI}$, confidence interval; TURP, transurethral resection of the prostate; IPSS, International Prostate Symptom Score; TUVP, transurethral vaporization of the prostate; HoLEP, holmium laser enucleation of the prostate; KTP, potassium-titanyl-phosphate.

\section{Late complications (Fig. 7)}

There were no statistically significant differences in any of the modalities compared with TURP. However, bladder neck contracture, urethral stricture, dysuria, and reintervention episode occurred more commonly in the KTP group than in the others (Table 5).

\section{DISCUSSION}

$\mathrm{BPH}$ results from the proliferation of smooth muscle cells and epithelial cells in the prostatic transitional zone [9]. The treatment of LUTS/BPH is aimed at improving QoL and preventing complications such as urinary tract infection and urinary retention. Treatment methods are largely divided into surgery, medi-

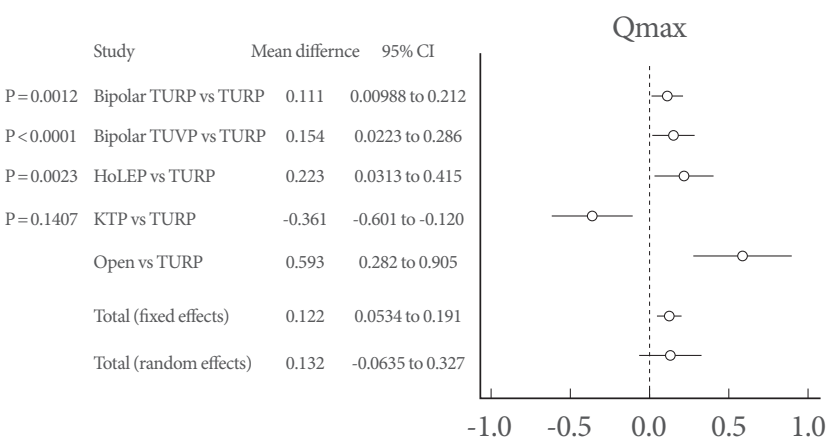

Fig. 3. Forest plot of maximum flow rate (Qmax). CI, confidence interval; TURP, transurethral resection of the prostate; IPSS, International Prostate Symptom Score; TUVP, transurethral vaporization of the prostate; HoLEP, holmium laser enucleation of the prostate; KTP, potassium-titanyl-phosphate.

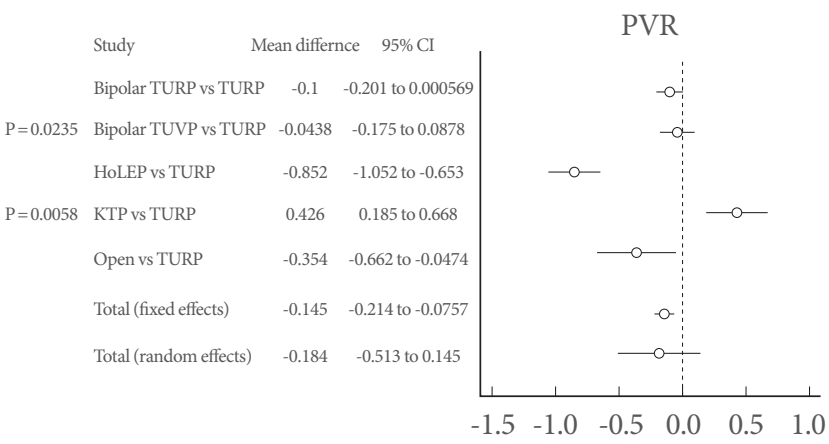

Fig. 4. Forest plot of postvoid residual urine volume (PVR). CI, confidence interval; TURP, transurethral resection of the prostate; IPSS, International Prostate Symptom Score; TUVP, transurethral vaporization of the prostate; HoLEP, holmium laser enucleation of the prostate; KTP, potassium-titanyl-phosphate. 
cal treatment, and watchful waiting [10]. Watchful waiting is applicable to patients with mild symptoms or with moderateto-severe symptoms but no complications. This treatment option requires measurement of prostate volume and Qmax annually [11]. For medical management, representative drugs are 5-ARIs and alpha-adrenergic blockers. 5-ARI lowers the serum dihydrotestosterone level and reduces the volume of the prostate [12]. Alpha-adrenergic blockers improve voiding symptoms by relaxation of smooth muscle in the prostate [13]. However, when patients have concomitant complications such as hematuria, infection, or urinary tract obstruction, surgical management is warranted [14]. Many surgical techniques have been

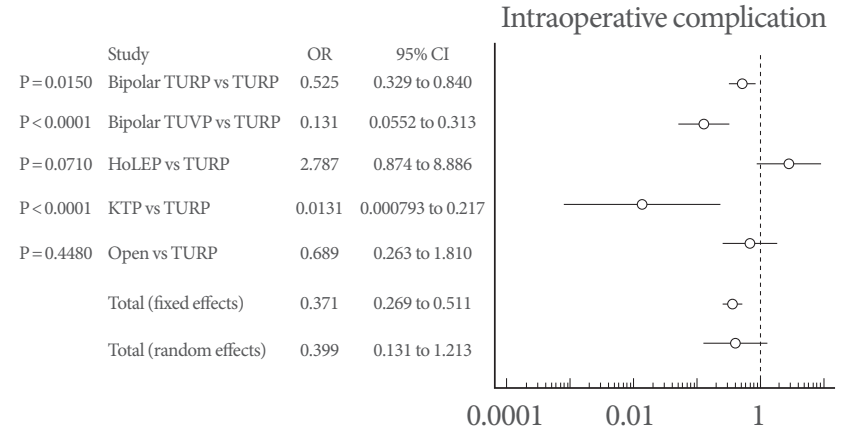

Fig. 5. Forest plot of intraoperative complications. TURP, transurethral resection of the prostate; $\mathrm{OR}$, odds ratio; $\mathrm{CI}$, confidence interval; TUVP, transurethral vaporization of the prostate; HoLEP, holmium laser enucleation of the prostate; KTP, potassiumtitanyl-phosphate.

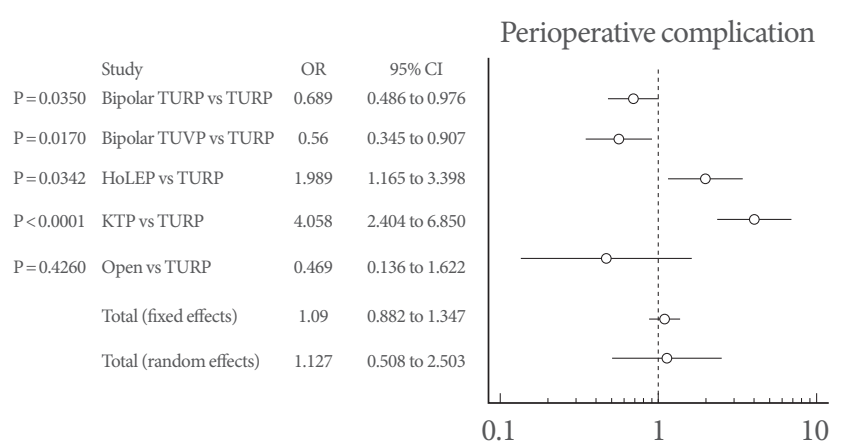

Fig. 6. Forest plot of perioperative complications. OR, odds ratio; $\mathrm{CI}$, confidence interval; TURP, transurethral resection of the prostate; TUVP, transurethral vaporization of the prostate; HoLEP, holmium laser enucleation of the prostate; KTP, potassium-titanyl-phosphate.

Table 3. Intraoperative complications

\begin{tabular}{lccccccc}
\hline Procedure & Bleeding & $\begin{array}{c}\text { Capsular } \\
\text { perforation }\end{array}$ & $\begin{array}{c}\text { Conversion } \\
\text { to TURP }\end{array}$ & $\begin{array}{c}\text { Bladder } \\
\text { mucosal injury }\end{array}$ & Transfusion & $\begin{array}{c}\text { TUR } \\
\text { syndrome }\end{array}$ & $\begin{array}{c}\text { Total } \\
\text { TURP (\%) }\end{array}$ \\
\hline Bipolar TURP (\%) & 1.7 & 1.8 & 0 & 0 & 4.3 & 0.9 & 8.7 \\
Bipolar TUVP (\%) & 0.6 & 1.5 & 0 & 0 & 2 & 0 & 5.5 \\
HoLEP (\%) & 0 & 0.4 & 0 & 0 & 0.6 & 0 & 1.6 \\
KTP (\%) & 0 & 0.2 & 0 & 2.6 & 0 & 0 & 2.8 \\
\hline
\end{tabular}

TURP, transurethral resection of prostate; TUVP, transurethral vaporisation of the prostate; HoLEP, holmium laser enucleation of the prostate; KTP, potassium-titanyl-phosphate.

Table 4. Perioperative complication

\begin{tabular}{lccccccccc}
\hline Procedure & AUR & $\begin{array}{c}\text { Clot } \\
\text { retention }\end{array}$ & $\begin{array}{c}\text { Secondary } \\
\text { apical } \\
\text { resection }\end{array}$ & $\begin{array}{c}\text { Secondary } \\
\text { coagulation }\end{array}$ & $\begin{array}{c}\text { Delay } \\
\text { bleeding }\end{array}$ & $\begin{array}{c}\text { Episodes of } \\
\text { hematuria }\end{array}$ & Urosepsis & UTI, fever & Total \\
\hline TURP (\%) & 3.7 & 4.5 & 0.1 & 0.6 & 0.9 & 3.8 & 0.1 & 3.8 & 17.5 \\
Bipolar TURP (\%) & 3.3 & 2.6 & 0 & 0 & 0.8 & 1.5 & 0 & 2.2 & 10.4 \\
Bipolar TUVP (\%) & 3.9 & 2.4 & 0 & 0 & 0.9 & 0 & 0 & 1.3 \\
HoLEP (\%) & 4.6 & 0 & 0.4 & 1.1 & 0 & 0 & 0 & 0.6 & 6.5 \\
KTP (\%) & 5.5 & 0 & 1.2 & 0 & 0.4 & 0 & 0 & 6.7 \\
\hline
\end{tabular}

AUR, acute urinary retention; UTI, urinary tract infection; TURP, transurethral resection of prostate; TUVP, transurethral vaporisation of the prostate; HoLEP, holmium laser enucleation of the prostate; KTP, potassium-titanyl-phosphate. 
Table 5. Late operative complication

\begin{tabular}{lcccccccc}
\hline Procedure & $\begin{array}{c}\text { Bladder neck } \\
\text { contracture }\end{array}$ & $\begin{array}{c}\text { Urethral } \\
\text { stricture }\end{array}$ & $\begin{array}{c}\text { Reinterven- } \\
\text { tion }\end{array}$ & $\begin{array}{c}\text { Secondary } \\
\text { treatment }\end{array}$ & $\begin{array}{c}\text { Transient } \\
\text { dysuria }\end{array}$ & Urgency & $\begin{array}{c}\text { Stress urinary } \\
\text { incontinence }\end{array}$ & Total \\
\hline TURP (\%) & 2.0 & 3.7 & 1.5 & 0.6 & 4.8 & 2.5 & 0.9 & 16.0 \\
Bipolar TURP (\%) & 1.2 & 3.0 & 1.2 & 1.2 & 2.3 & 1.2 & 0.3 & 10.4 \\
Bipolar TUVP (\%) & 0.7 & 2.6 & 1.5 & 0.6 & 5.2 & 1.9 & 0.2 & 12.7 \\
HoLEP (\%) & 1.6 & 4.0 & 0.9 & 0 & 0.9 & 4.4 & 0.9 & 12.7 \\
KTP (\%) & 5.9 & 4.7 & 6.7 & 0 & 15.7 & 0 & 0.7 & 33.7 \\
\hline
\end{tabular}

TURP, transurethral resection of prostate; TUVP, transurethral vaporisation of the prostate; HoLEP, holmium laser enucleation of the prostate; KTP, potassium-titanyl-phosphate.

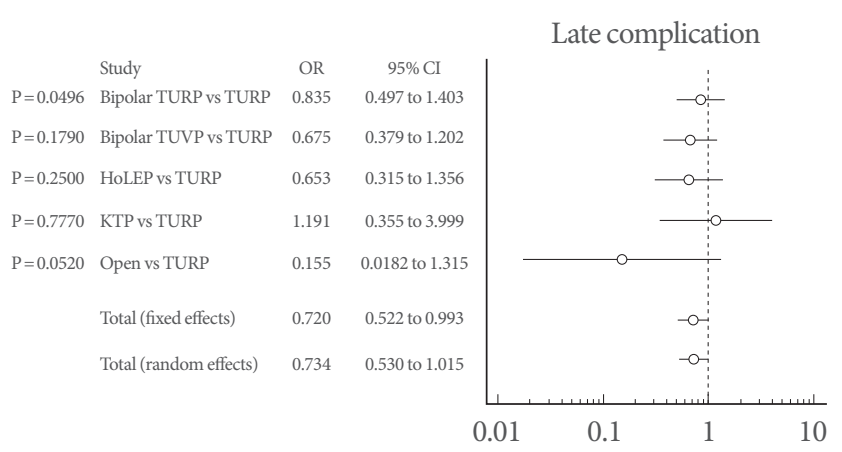

Fig. 7. Forest plot of late complications. OR, odds ratio; CI, confidence interval; TURP, transurethral resection of the prostate; TUVP, transurethral vaporization of the prostate; HoLEP, holmium laser enucleation of the prostate; KTP, potassium-titanylphosphate.

developed, such as open prostatectomy, HoLEP, KTP, TURP, bipolar TURP, and bipolar TUVP. Until now, TURP has remained the gold standard treatment option for LUTS/BPH [11]. However, TURP faces many challenges, such as morbidity and functional outcomes [15].

\section{TURP}

Accord to our results, TURP was one of the most effective surgical modalities for relieving the obstruction due to BPH. This method needed less reintervention and showed effectiveness for relieving the voiding symptoms, including reduction of IPSS and improvement of Qmax. However, as shown in Tables 3 and 4, TURP has many intraoperative and perioperative complications, such as bleeding, clot retention, and transfusion. Nonetheless, the late complication rate was not inferior compared with other surgical methods. Considering these results, TURP is a worthy mode of surgical intervention for treating LUTS/BPH.

\section{Bipolar TURP}

In our meta-analysis, bipolar TURP showed comparable functional outcomes to TURP. Moreover, bipolar TURP showed a shorter length of Foley catheterization and lower intraoperative and perioperative complications. However, to replace TURP, longterm, large-scale, randomized controlled trials will be needed.

\section{HoLEP}

Many articles have shown that for large prostates, HoLEP shows superior functional outcomes to open prostatectomy [16,17]. Some parameters of HoLEP, such as length of hospital stay and duration of Foley catheterization, showed supremacy to TURP. Moreover, HoLEP seemed to be effective for avoiding intraoperative and perioperative complications, even though it showed bladder mucosal injury during morcellation of resected prostate adenoma. To confirm the effectiveness and safety of HoLEP, many studies should be performed in relatively small prostates, and long-term comparative studies are needed.

\section{KTP Laser Vaporization of the Prostate}

For small to moderate prostate volume, KTP showed comparable functional outcomes, such as for Qmax, PVR, IPSS, and QoL, to TURP. Also, KTP had some benefits, such as a lower incidence of intraoperative complications compared with TURP in patients with a small to moderate prostate volume. However, KTP showed a higher incidence of late complications, such as bladder neck contracture and urethral stricture. Thus, long-term, large-scale randomized controlled studies, conducted for large prostates, should be conducted to prove the usefulness and safety of KTP.

We conducted a meta-analysis of transurethral surgeries for LUTS/BPH. It will be helpful to identify the advantages and disadvantages of each surgical modality. However, we conducted the meta-analysis without a quality analysis of the articles. This is a limitation of the present study even though we per- 
formed our analysis of the articles that showed the highest level of evidence.

In conclusion, this study showed statistically comparable efficacy and overall morbidity of transurethral surgeries compared with TURP. Functional outcomes and complications at each step varied for each modality. The selection of an appropriate surgical modality for BPH should be assessed by fully understanding each patient's clinical conditions.

\section{CONFLICT OF INTEREST}

No potential conflict of interest relevant to this article was reported.

\section{SUPPLEMENTARY MATERIALS}

Supplementary material can be found via http://www.einj.org/ src/sm/inj-17-59-s001.pdf.

\section{REFERENCES}

1. Lepor H. Pathophysiology, epidemiology, and natural history of benign prostatic hyperplasia. Rev Urol 2004;6 Suppl 9:S3-10.

2. Hoke GP, McWilliams GW. Epidemiology of benign prostatic hyperplasia and comorbidities in racial and ethnic minority populations. Am J Med 2008;121(8 Suppl 2):S3-10.

3. Speakman MJ. Who should be treated and how? Evidence-based medicine in symptomatic BPH. Eur Urol 1999;36 Suppl 3:40-51.

4. Souverein PC, Erkens JA, de la Rosette JJ, Leufkens HG, Herings RM. Drug treatment of benign prostatic hyperplasia and hospital admission for BPH-related surgery. Eur Urol 2003;43:528-34.

5. Rassweiler J, Teber D, Kuntz R, Hofmann R. Complications of transurethral resection of the prostate (TURP): incidence, management, and prevention. Eur Urol 2006;50:969-79.

6. Ahyai SA, Gilling P, Kaplan SA, Kuntz RM, Madersbacher S, Montorsi F, et al. Meta-analysis of functional outcomes and complications following transurethral procedures for lower urinary tract symptoms resulting from benign prostatic enlargement. Eur Urol 2010;58:384-97.
7. Chung JH, Kang DH, Jo JK, Lee SW. Assessing the quality of randomized controlled trials published in the Journal of Korean Medical Science from 1986 to 2011. J Korean Med Sci 2012;27:973-80.

8. Higgins JP, Thompson SG, Deeks JJ, Altman DG. Measuring inconsistency in meta-analyses. BMJ 2003;327:557-60.

9. Auffenberg GB, Helfand BT, McVary KT. Established medical therapy for benign prostatic hyperplasia. Urol Clin North Am 2009;36: 443-59.

10. Madersbacher S, Alivizatos G, Nordling J, Sanz CR, Emberton M, de la Rosette JJ. EAU 2004 guidelines on assessment, therapy and follow-up of men with lower urinary tract symptoms suggestive of benign prostatic obstruction (BPH guidelines). Eur Urol 2004;46: 547-54.

11. McVary KT, Roehrborn CG, Avins AL, Barry MJ, Bruskewitz RC, Donnell RF, et al. Update on AUA guideline on the management of benign prostatic hyperplasia. J Urol 2011;185:1793-803.

12. Marberger M, Harkaway R, de la Rosette J. Optimising the medical management of benign prostatic hyperplasia. Eur Urol 2004;45: 411-9.

13. Chapple CR. Alpha adrenoceptor antagonists in the year 2000: is there anything new? Curr Opin Urol 2001;11:9-16.

14. Taylor J, Harrison SC, Assassa RP, McGrother CW; Leicestershire MRC Incontinence Study Group. The pattern and progression of lower urinary tract symptoms after transurethral prostatectomy compared with those seen in the general population. Eur Urol 2007; 51:1023-9.

15. Mebust WK, Holtgrewe HL, Cockett AT, Peters PC. Transurethral prostatectomy: immediate and postoperative complications. A cooperative study of 13 participating institutions evaluating 3,885 patients. J Urol 1989;141:243-7.

16. Naspro R, Suardi N, Salonia A, Scattoni V, Guazzoni G, Colombo R, et al. Holmium laser enucleation of the prostate versus open prostatectomy for prostates $>70 \mathrm{~g}$ : 24-month follow-up. Eur Urol 2006; 50:563-8.

17. Kuntz RM, Lehrich K. Transurethral holmium laser enucleation versus transvesical open enucleation for prostate adenoma greater than $100 \mathrm{gm} .::$ a randomized prospective trial of 120 patients. J Urol 2002;168(4 Pt 1):1465-9. 